\title{
New solvable many-body problems in the plane
}

F CALOGERO ${ }^{a}$ and J-P FRANCOISE ${ }^{b}$

${ }^{a}$ Dipartimento di Fisica, Università di Roma "La Sapienza", 00185 Roma, Italy; Istituto Nazionale di Fisica Nucleare, Sezione di Roma

E-mail: francesco.calogero@roma1.infn.it

${ }^{b}$ Laboratoire J.-L. Lions, Université P.-M. Curie, Paris VI and CNRS, 175 Rue de Chevaleret, 75013 Paris, France

E-mail: jpf@ccr.jussieu.fr

Received July 6, 2005; Accepted September 3, 2005

\begin{abstract}
We revisit an integrable (indeed, superintegrable and solvable) many-body model introduced almost two decades ago by Gibbons and Hermsen and by Wojciechowski, and we modify it so that its generic solutions are all isochronous (namely, completely periodic with fixed period). We then show how this model (or rather the more basic dynamical system that underlies its solvable character, and other avatars of it) can be conveniently reinterpreted as (rotation-invariant) models in the plane; and we thereby present several new (solvable, isochronous and rotation-invariant) many-body problems in the plane.
\end{abstract}

\section{Introduction and main results}

Almost two decades ago J. Gibbons and T. Hermsen [16], and almost simultaneously (and certainly independently) S. Wojciechowski [23], discussed the integrable (indeed solvable) dynamical system the equations of motion of which we write here as follows:

$$
\begin{aligned}
& \ddot{z}_{n}=-2 \sum_{m=1, m \neq n}^{N} \frac{g_{n m} g_{m n}}{\left(z_{n}-z_{m}\right)^{3}}, \\
& \dot{g}_{n m}=-\sum_{\ell=1, \ell \neq m, n}^{N} g_{n \ell} g_{\ell m}\left(\frac{1}{\left(z_{n}-z_{\ell}\right)^{2}}-\frac{1}{\left(z_{m}-z_{\ell}\right)^{2}}\right), \quad n \neq m .
\end{aligned}
$$

Here we consider the $N$ evolving quantities $z_{n} \equiv z_{n}(t)$ as the main dynamical variables, and the $N(N-1)$ evolving quantities $g_{n m} \equiv g_{n m}(t), n \neq m$, as auxiliary dependent variables; the rest of the notation is, we trust, self-evident: in particular, superimposed dots always denote differentiation with respect to the (real) independent variable $t$ ("time"), and indices such as $n, m$ always run from 1 to $N$ (unless otherwise explicitly indicated).

Copyright (C) 2006 by Calogero F and Françoise J-P 
This model was introduced [16] [23] as a variation, characterized by additional "internal" degrees of freedom (possibly representing spin) associated with the auxiliary dependent variables $g_{n m}$, of the well-known [18] [7] [10] integrable - indeed solvable [21] many-body problem on the line defined by the equations of motion (1.1a) without (1.1b) and with the quantities $g_{n m}$ time-independent and all equal (say: $g_{n m}=i c, \dot{c}=0$ ). These models, (1.1), as well as analogous ones, have also been investigated, earlier and later, in rather different or quite analogous contexts, see for instance [3] [22] [25] [24] [26] [19] [4] [5] [17] [20] [2] [1] (and additional references cited in these papers). One of the models we consider here is merely a marginally more general version of the equations of motion (1.1), namely

$$
\begin{aligned}
\ddot{z}_{n}+a \dot{z}_{n}+b z_{n}= & -2 \sum_{m=1, m \neq n}^{N} \frac{g_{n m} g_{m n}}{\left(z_{n}-z_{m}\right)^{3}}, \\
\dot{g}_{n m}+a g_{n m}= & -\sum_{\substack{\ell=1, \ell \neq m, n\\
}}^{N} g_{n \ell} g_{\ell m}\left(\frac{1}{\left(z_{n}-z_{\ell}\right)^{2}}-\frac{1}{\left(z_{m}-z_{\ell}\right)^{2}}\right) \\
& +g_{n m}\left(f_{n}-f_{m}\right), \quad n \neq m .
\end{aligned}
$$

Note the additional terms in the left-hand sides proportional to the two constants $a$ and $b$, and the appearance in the right-hand side of $(1.2 \mathrm{~b})$ of the source terms $f_{n}$, which can be assigned as arbitrary functions of the time $t, f_{n} \equiv f_{n}(t)$, or as arbitrary functions of the dependent variables $z_{n}(t)$ and $g_{n m}(t)$, without spoiling the solvability of the model, namely the possibility to reduce its solution to a sequence of purely algebraic operations (to get the main dependent variables $z_{n}(t)$; and moreover a quadrature, to get the auxiliary dependent variables $\left.g_{n m}(t)\right)$. Actually the presence of the quantities $f_{n}$ in $(1.2 \mathrm{~b})$ could be eliminated via the "similarity transformation"

$$
g_{n m}(t)=\exp \left[F_{n}(t)-F_{m}(t)\right] \check{g}_{n m}(t)
$$

with

$$
\dot{F}_{n}(t)=f_{n}(t)
$$

which clearly entails that the equations of motion (1.2) become

$$
\begin{aligned}
& \ddot{z}_{n}+a \dot{z}_{n}+b z_{n}=-2 \sum_{m=1, m \neq n}^{N} \frac{\check{g}_{n m} \check{g}_{m n}}{\left(z_{n}-z_{m}\right)^{3}}, \\
& \dot{\check{g}}_{n m}+a \check{g}_{n m}=-\sum_{\ell=1, \ell \neq m, n}^{N} \check{g}_{n \ell} \check{g}_{\ell m}\left(\frac{1}{\left(z_{n}-z_{\ell}\right)^{2}}-\frac{1}{\left(z_{m}-z_{\ell}\right)^{2}}\right), \quad n \neq m .
\end{aligned}
$$

An advantage of this simpler version of the equations of motion is to allow the reduction $\check{g}_{n m}=-\check{g}_{m n}$ (since clearly $(1.4 \mathrm{~b})$ entails that validity of this restriction at the initial 
time, $\check{g}_{n m}(0)=-\check{g}_{m n}(0)$ implies its validity for all time, $\left.\check{g}_{n m}(t)=-\check{g}_{m n}(t)\right)$, yielding the reduced system

$$
\begin{aligned}
\ddot{z}_{n}+a \dot{z}_{n}+b z_{n} & =2 \sum_{m=1, m \neq n}^{N} \frac{\check{g}_{n m}^{2}}{\left(z_{n}-z_{m}\right)^{3}}, \quad \check{g}_{n m}^{2}=\check{g}_{n m}^{2}, \\
\dot{g}_{n m}+a \check{g}_{n m} & =-\sum_{\ell=1, \ell \neq m, n}^{N} \check{g}_{n \ell} \check{g}_{\ell m}\left(\frac{1}{\left(z_{n}-z_{\ell}\right)^{2}}-\frac{1}{\left(z_{m}-z_{\ell}\right)^{2}}\right), \\
\check{g}_{n m} & =-\check{g}_{m n}, \quad n \neq m .
\end{aligned}
$$

On the other hand the version (1.2) allows reformulations obtained by assigning a specific expression of $f_{n}$ in terms of the dependent variables: for instance via the assignment

$$
f_{n}=\breve{f}_{n}+\sum_{\ell=1, \ell \neq n}^{N} \frac{g_{n \ell}}{\left(z_{n}-z_{\ell}\right)^{2}}
$$

the evolution equations $(1.2 \mathrm{~b})$ become

$$
\begin{gathered}
\dot{g}_{n m}+a g_{n m}=\sum_{\ell=1 ; \ell \neq n, m}^{N} \frac{g_{n m} g_{n \ell}-g_{n \ell} g_{\ell m}}{\left(z_{n}-z_{\ell}\right)^{2}} \\
-\sum_{\ell=1 ; \ell \neq n, m}^{N} \frac{g_{n m} g_{m \ell}-g_{n \ell} g_{\ell m}}{\left(z_{m}-z_{\ell}\right)^{2}} \\
+g_{n m} \frac{\left(g_{n m}-g_{m n}\right)}{\left(z_{m}-z_{m}\right)^{2}}+g_{n m}\left(\breve{f}_{n}-\breve{f}_{m}\right), \quad n \neq m,
\end{gathered}
$$

admitting as a subcase (see (1.2a)), for $a=0$ and $\breve{f}_{n}=0$, the original model [18] [7] [10] with the quantities $g_{n m}$ time-independent and all equal, $g_{n m}=i c, \dot{c}=0$. But note that, except in this special case, these equations of motion, (1.6b), as indeed generally the equations of motion (1.2b) whenever the quantities $f_{n}$ are effectively present, are incompatible with the assumption that the quantities $g_{n m}$ have a definite symmetry (even or odd) under the exchange of the two indices $n, m$.

The first main finding of this paper is to point out the remarkable properties of the model (1.2) with the special assignment

$$
a=-i \mu \omega, \quad b=\frac{1}{4}\left(1-\mu^{2}\right) \omega^{2},
$$

entailing that its equations of motion read

$$
\begin{aligned}
\ddot{z}_{n}-i \mu \omega \dot{z}_{n}+\frac{1}{4}\left(1-\mu^{2}\right) \omega^{2} z_{n}=-2 \sum_{m=1, m \neq n}^{N} \frac{g_{n m} g_{m n}}{\left(z_{n}-z_{m}\right)^{3}}, \\
\dot{g}_{n m}-i \mu \omega g_{n m}=-\sum_{\substack{\ell=1, \ell \neq m, n \\
\\
+}} g_{n m}\left(f_{n}-f_{m}\right), \quad n \neq m,
\end{aligned}
$$


and likewise that (1.6b) becomes

$$
\begin{gathered}
\dot{g}_{n m}-i \mu \omega g_{n m}=\sum_{\ell=1 ; \ell \neq n, m}^{N} \frac{g_{n m} g_{n \ell}-g_{n \ell} g_{\ell m}}{\left(z_{n}-z_{\ell}\right)^{2}} \\
\sum_{\ell=1 ; \ell \neq n, m}^{N} \frac{g_{n m} g_{m \ell}-g_{n \ell} g_{\ell m}}{\left(z_{m}-z_{\ell}\right)^{2}} \\
+g_{n m} \frac{\left(g_{n m}-g_{m n}\right)}{\left(z_{m}-z_{m}\right)^{2}}+g_{n m}\left(\breve{f}_{n}-\breve{f}_{m}\right), \quad n \neq m .
\end{gathered}
$$

Hereafter (except when a different hypothesis is explicitly made, see below) we assume that the constant $\omega$ is real (in fact, without loss of generality, positive, $\omega>0$ ), and we associate to it the period

$$
T=\frac{\pi}{\omega} .
$$

As for the constant $\mu$, we assume hereafter that it is a rational number,

$$
\mu=\frac{p}{q}
$$

with $p$ and $q$ two coprime integers and $q>0$. We then show that, under these conditions, the generic solution - which is obtained in Section 2 - of these equations of motion, (1.8), is isochronous - namely, completely periodic with a fixed period largely independent of the initial data - a period $\tilde{T}$ which is a simple integer multiple of $q T$ - at least for the main dependent variables:

$$
z_{n}(t+\tilde{T})=z_{n}(t) .
$$

This outcome obtains for any assignment of the time evolution of the source terms $f_{n}(t)$; indeed it turns out - remarkably, or perhaps obviously, given the possibility to transform (1.2) into (1.4) via (1.3) - that the time evolution of the main dependent variables $z_{n}(t)$ is completely independent of the assignment of the source terms $f_{n}(t)$. The time evolution of the auxiliary variables $g_{n m}(t)$ is instead affected by the time-evolution of the source terms $f_{n}(t)$, hence it may itself display properties of periodicity only if the source terms satisfy themselves appropriate periodicity properties - as entailed by the solution of the problem described in Section 2.

Let us insert here a remark which is applicable to the dynamical systems we just presented, (1.8) and (1.9), but obviously as well to many others of the systems considered herein as well as to other ones. Clearly these systems can be generalized by adding to the right-hand side of (1.8a) an arbitrary (possibly time-dependent) "external force" $f(t)$, the only effect of which is to transform the solutions according to the rule $z_{n}(t) \rightarrow z_{n}(t)+z_{0}(t)$ with $z_{0}(t)$ defined by the following ODE and initial conditions:

$$
\ddot{z}_{0}-i \mu \omega \dot{z}_{0}+\frac{1}{4}\left(1-\mu^{2}\right) \omega^{2} z_{0}=f(t), \quad z_{0}(0)=\dot{z}_{0}(0)=0 .
$$

The only effect of $z_{0}(t)$ is to cause a corresponding transformation, $Z(t) \rightarrow Z(t)+z_{0}(t)$, of the center of mass $Z(t)=\frac{1}{N} \sum_{n=1}^{N} z_{n}(t)$ of the system. Note that this entails that, 
when the force $f(t)$ has a periodicity at resonance with one of those characteristic of the solution of the homogeneous part of this ODE, (1.13), say $f(t)=a \sin [(\mu+1) \omega t+\theta]$ or $f(t)=a \sin [(\mu-1) \omega t+\theta]$ (with $a$ and $\theta$ arbitrary constants), then the center of mass $Z(t)$ (and, with it, all the coordinates $z_{n}(t)$ ) escapes asymptotically to infinity (namely, $Z(t)$ diverges, linearly in $t$, as $t \rightarrow \infty)$.

The equations of motion (1.8) appear particularly interesting in the special case $\mu=0$, when they become real. But, even in this case (and by necessity whenever instead $\mu \neq 0$ ), it is more interesting to focus attention on the solutions of (1.8) in the complex domain, not only because their behavior is then much richer, but as well because, by identifying in the standard manner (see, for instance, [9] [10], and the relations (3.49) below) the complex plane with the real physical plane, these equations of motion, (1.8), can be reformulated as the following real equations characterizing the motion of particles in the plane:

$$
\begin{aligned}
& \ddot{\vec{r}}_{n}-\mu \omega \hat{k} \wedge \dot{\vec{r}}_{n}+\frac{1}{4}\left(1-\mu^{2}\right) \omega^{2} \vec{r}_{n} \\
& =-2 \sum_{m=1, m \neq n}^{N} r_{n m}^{-8} \vec{V}^{(7)}\left(\vec{\rho}_{n m}, \vec{\rho}_{n m}, \vec{\rho}_{m n}, \vec{\rho}_{m n} ; \vec{r}_{n m}, \vec{r}_{n m}, \vec{r}_{n m}\right), \\
& \quad \dot{\vec{\rho}}_{n m}-\frac{1}{2} \mu \omega \hat{k} \wedge \vec{\rho}_{n m} \\
& =-\frac{1}{2} \rho_{n m}^{-2} \sum_{\ell=1, \ell \neq m, n}^{N}\left[\frac{\vec{V}^{(7)}\left(\vec{\rho}_{n \ell}, \vec{\rho}_{n \ell}, \vec{\rho}_{\ell m}, \vec{\rho}_{\ell m} ; \vec{\rho}_{n m}, \vec{r}_{n \ell}, \vec{r}_{n \ell}\right)}{r_{n \ell}^{4}}\right. \\
& \left.\quad-\frac{\vec{V}^{(7)}\left(\vec{\rho}_{n \ell}, \vec{\rho}_{n \ell}, \vec{\rho}_{\ell m}, \vec{\rho}_{\ell m} ; \vec{\rho}_{n m}, \vec{r}_{m \ell}, \vec{r}_{m \ell}\right)}{r_{m \ell}^{4}}\right] \\
& +\vec{\rho}_{n m}\left(f_{n}^{(1)}-f_{m}^{(1)}\right)+\hat{k} \wedge \vec{\rho}_{n m}\left(f_{n}^{(2)}-f_{m}^{(2)}\right), \quad n \neq m,
\end{aligned}
$$

and likewise (1.9) can be reformulated as follows:

$$
\begin{aligned}
& \dot{\vec{\rho}}_{n m}-\frac{1}{2} \mu \omega \hat{k} \wedge \vec{\rho}_{n m} \\
= & \frac{1}{2} \rho_{n m}^{-2} \sum_{\ell=1, \ell \neq m, n}^{N}\left\{\left[\frac{\vec{V}^{(7)}\left(\vec{\rho}_{n \ell}, \vec{\rho}_{n \ell}, \vec{\rho}_{n m}, \vec{\rho}_{n m} ; \vec{\rho}_{n m}, \vec{r}_{n \ell}, \vec{r}_{n \ell}\right)}{r_{n \ell}^{4}}\right.\right. \\
& \left.-\frac{\vec{V}^{(7)}\left(\vec{\rho}_{n \ell}, \vec{\rho}_{n \ell}, \vec{\rho}_{\ell m}, \vec{\rho}_{\ell m} ; \vec{\rho}_{n m}, \vec{r}_{n \ell}, \vec{r}_{n \ell}\right)}{r_{n \ell}^{4}}\right] \\
& -\left[\frac{\vec{V}^{(7)}\left(\vec{\rho}_{n \ell}, \vec{\rho}_{n \ell}, \vec{\rho}_{n m}, \vec{\rho}_{n m} ; \vec{\rho}_{n m}, \vec{r}_{m \ell}, \vec{r}_{m \ell}\right)}{r_{m \ell}^{4}}\right. \\
& \left.\left.-\frac{\vec{V}^{(7)}\left(\vec{\rho}_{n \ell}, \vec{\rho}_{n \ell}, \vec{\rho}_{\ell m}, \vec{\rho}_{\ell m} ; \vec{\rho}_{n m}, \vec{r}_{m \ell}, \vec{r}_{m \ell}\right)}{r_{m \ell}^{4}}\right]\right\} \\
& +\vec{\rho}_{n m}\left(\breve{f}_{n}^{(1)}-\breve{f}_{m}^{(1)}\right)+\hat{k} \wedge \vec{\rho}_{n m}\left(\breve{f}_{n}^{(2)}-\breve{f}_{m}^{(2)}\right), \quad n \neq m .
\end{aligned}
$$

Here everything is now real, superimposed arrows denote two-vectors in the horizontal plane (assumed for convenience embedded in ordinary, three-dimensional, space, say $\vec{r} \equiv$ 
$(x, y, 0))$, the main dependent variables are the $N$ two-vectors $\vec{r}_{n} \equiv \vec{r}_{n}(t)$, the auxiliary dependent variables are the $N(N-1)$ two-vectors $\vec{\rho}_{n m} \equiv \vec{\rho}_{n m}(t)$, the symbol $\hat{k}$ denotes the vertical unit vector $\hat{k} \equiv(0,0,1)$ so that $\hat{k} \wedge \vec{r} \equiv(-y, x, 0)$ (see (3.49)), the $2 N$ scalar source terms $f_{n}^{(1)} \equiv f_{n}^{(1)}(t), f_{n}^{(2)} \equiv f_{n}^{(2)}(t)$ are arbitrary, and we use (here, and often as well below) the short-hand notation

$$
\vec{r}_{n m} \equiv \vec{r}_{n}-\vec{r}_{m}
$$

entailing of course $r_{n m}^{2}=r_{n}^{2}+r_{m}^{2}-2 \vec{r}_{n} \cdot \vec{r}_{m}$, and the two-vector $\vec{V}^{(7)}$ is defined in terms of its 7 two-vector arguments in the Appendix, see (A.13) - but note the simplifications entailed by the equality of several of its arguments in these equations of motion (1.14) and (1.15), so that for instance the vector $\vec{V}^{(7)}$ appearing in the right -hand side of (1.14a) can be written as follows:

$$
\begin{aligned}
& \vec{V}^{(7)}\left(\vec{\rho}_{n m}, \vec{\rho}_{n m}, \vec{\rho}_{m n}, \vec{\rho}_{m n} ; \vec{r}_{n m}, \vec{r}_{n m}, \vec{r}_{n m}\right) \\
&=a_{n m} \vec{r}_{n m}+b_{n m} \vec{\rho}_{n m}+b_{m n} \vec{\rho}_{m n}, \\
& a_{n m}= 16\left(\vec{r}_{n m} \cdot \vec{\rho}_{n m}\right)^{2}\left(\vec{r}_{n m} \cdot \vec{\rho}_{m n}\right)^{2} \\
&-2 r_{n m}^{2}\left[3 \rho_{n m}^{2}\left(\vec{r}_{n m} \cdot \vec{\rho}_{m n}\right)^{2}+3 \rho_{m n}^{2}\left(\vec{r}_{n m} \cdot \vec{\rho}_{n m}\right)^{2}\right. \\
&\left.+2\left(\vec{r}_{n m} \cdot \vec{\rho}_{n m}\right)\left(\vec{r}_{n m} \cdot \vec{\rho}_{m n}\right)\left(\vec{\rho}_{n m} \cdot \vec{\rho}_{m n}\right)\right]+r_{n m}^{4} \rho_{n m}^{2} \rho_{m n}^{2}, \\
& b_{n m}=-2 r_{n m}^{2}\left(\vec{r}_{n m} \cdot \vec{\rho}_{n m}\right)\left[2\left(\vec{r}_{n m} \cdot \vec{\rho}_{m n}\right)^{2}-r_{n m}^{2} \rho_{m n}^{2}\right] .
\end{aligned}
$$

As for the rest of the notation, we hope it is self-evident, otherwise see Section 3 where the equivalence is demonstrated of this (real) many-body problem in the horizontal plane (1.14) with the (complex) system (1.8).

Note that these equations of motion become somewhat neater in the reduced case (1.5) (which implies the consistent if somewhat awkward restriction $\vec{\rho}_{n m}=\hat{k} \wedge \vec{\rho}_{m n}$; see (3.55) and (3.57) in Section 3), when for instance the first set, (1.14a), read

$$
\begin{array}{r}
\ddot{\vec{r}}_{n}-\mu \omega \hat{k} \wedge \dot{\vec{r}}_{n}+\frac{1}{4}\left(1-\mu^{2}\right) \omega^{2} \vec{r}_{n}=8 \sum_{m=1, m \neq n}^{N} r_{n m}^{-8} . \\
\cdot\left[\vec{r}_{n m}\left\{\left(r_{n m}^{2} \rho_{n m}^{2}\right)^{2}-2\left(\vec{r}_{n m} \cdot \vec{\rho}_{m n}\right)^{2}\left(r_{n m}^{2} \rho_{m n}^{2}\right)+2\left(\vec{r}_{n m} \cdot \vec{\rho}_{m n}\right)^{4}\right\}\right. \\
+\hat{k} \wedge \vec{r}_{n m}\left\{\left(\vec{r}_{n m} \cdot \vec{\rho}_{n m}\right)\left(\hat{k} \cdot \vec{r}_{n m} \wedge \vec{\rho}_{n m}\right) .\right. \\
\left.\left.\cdot\left[2\left(\vec{r}_{n m} \cdot \vec{\rho}_{n m}\right)^{2}-r_{n m}^{2} \rho_{n m}^{2}\right]\right\}\right], \vec{\rho}_{n m}=\hat{k} \wedge \vec{\rho}_{m n} .
\end{array}
$$

Let us emphasize that these equations of motion (1.14) - the second main finding reported in this paper - are translation-invariant as far as the main dependent variables $\vec{r}_{n}$ are concerned (namely, they are invariant under the translation $\vec{r}_{n}(t) \Rightarrow \vec{r}_{n}(t)+\vec{r}_{0}$ with $\vec{r}_{0}$ an arbitrary constant two-vector, $\dot{\vec{r}}_{0}=0$ ) and, as it is obvious from their covariant structure, they are rotation-invariant - a nontrivial feature, which is of course essential for them to acquire physical relevance, at least as toy many-body models. 
As already mentioned, the solution of the initial-value problem for this many-body system in the plane - in its complex version (1.2) - is provided in the following Section 2 , while the relation of the complex version (1.2) to the real two-vector version (1.14) is provided in Section 3. But the results of these two sections also yield several other avatars of this model (or rather of the basic dynamical system that underlies its solvability), avatars which are obtained in their complex versions (including (1.8)) in Section 2 where the solution of the corresponding initial-value problems is also provided - and are then reformulated as real two-vector models in the plane in Section 3. We now display without much commentary these (rotation-invariant!) many-body problems in the horizontal plane. Their novelty, as well as their unified treatment, constitute the third main finding of this paper.

The equations of motion of the first of these models read

$$
\begin{aligned}
& \ddot{\vec{r}}_{n}-\mu \omega \hat{k} \wedge \dot{\vec{r}}_{n}+\frac{1}{4}\left(1-\mu^{2}\right) \omega^{2} \vec{r}_{n}=-2 \sum_{m=1, m \neq n}^{N} r_{n m}^{-4} \\
& {\left[\left(\lambda_{n m}^{(1)} \lambda_{m n}^{(1)}-\lambda_{n m}^{(2)} \lambda_{m n}^{(2)}\right) \vec{r}_{n m}+\left(\lambda_{n m}^{(1)} \lambda_{m n}^{(2)}+\lambda_{n m}^{(2)} \lambda_{m n}^{(1)}\right) \hat{k} \wedge \vec{r}_{n m}\right]} \\
& \dot{\lambda}_{n m}^{(1)}+\mu \omega \lambda_{n m}^{(2)}+2 \lambda_{n m}^{(2)} \frac{\vec{r}_{n m} \cdot \hat{k} \wedge \dot{\vec{r}}_{n m}}{r_{n m}^{2}} \\
& =\sum_{\ell=1 ; \ell \neq m, n}^{N}\left[\left(\lambda_{n \ell}^{(1)} \lambda_{\ell m}^{(1)}-\lambda_{n \ell}^{(2)} \lambda_{\ell m}^{(2)}\right)\left(-\frac{1}{r_{n \ell}^{2}}+\frac{1}{r_{m \ell}^{2}}\right)\right. \\
& \left.+\left(\lambda_{n \ell}^{(1)} \lambda_{\ell m}^{(2)}+\lambda_{n \ell}^{(2)} \lambda_{\ell m}^{(1)}\right) \frac{\vec{r}_{n m} \cdot \hat{k} \wedge\left(\vec{r}_{n \ell}+\vec{r}_{m \ell}\right)}{r_{n \ell}^{2} r_{m \ell}^{2}}\right] \\
& +\lambda_{n m}^{(1)}\left(f_{n}^{(1)}-f_{m}^{(1)}\right)-\lambda_{n m}^{(2)}\left(f_{n}^{(2)}-f_{m}^{(2)}\right), \quad n \neq m, \\
& \dot{\lambda}_{n m}^{(2)}-\mu \omega \lambda_{n m}^{(1)}+2 \lambda_{n m}^{(1)} \frac{\vec{r}_{n m} \cdot \hat{k} \wedge \dot{\vec{r}}_{n m}}{r_{n m}^{2}} \\
& =\sum_{\ell=1 ; \ell \neq m, n}^{N}\left[\left(\lambda_{n \ell}^{(1)} \lambda_{\ell m}^{(2)}+\lambda_{n \ell}^{(2)} \lambda_{\ell m}^{(1)}\right)\left(-\frac{1}{r_{n \ell}^{2}}+\frac{1}{r_{m \ell}^{2}}\right)\right. \\
& \left.-\left(\lambda_{n \ell}^{(1)} \lambda_{\ell m}^{(1)}-\lambda_{n \ell}^{(2)} \lambda_{\ell m}^{(2)}\right) \frac{\vec{r}_{n m} \cdot \hat{k} \wedge\left(\vec{r}_{n \ell}+\vec{r}_{m \ell}\right)}{r_{n \ell}^{2} r_{m \ell}^{2}}\right] \\
& +\lambda_{n m}^{(1)}\left(f_{n}^{(2)}-f_{m}^{(2)}\right)+\lambda_{n m}^{(2)}\left(f_{n}^{(1)}-f_{m}^{(1)}\right), \quad n \neq m .
\end{aligned}
$$

Here the $2 N(N-1)$ auxiliary variables $\lambda_{n m}^{(1)} \equiv \lambda_{n m}^{(1)}(t)$ and $\lambda_{n m}^{(2)} \equiv \lambda_{n m}^{(2)}(t)$ are of course real, and are to be treated as scalars. 
The equations of motion of the second of these models read

$$
\begin{aligned}
& \ddot{\vec{r}}_{n}-\mu \omega \hat{k} \wedge \dot{\vec{r}}_{n}+\frac{1}{4}\left(1-\mu^{2}\right) \omega^{2} \vec{r}_{n}=2 \sum_{m=1, m \neq n}^{N} r_{n m}^{-2} \\
& {\left[\vec{\chi}_{n m}\left(\vec{\chi}_{m n} \cdot \vec{r}_{n m}\right)+\vec{\chi}_{m n}\left(\vec{\chi}_{n m} \cdot \vec{r}_{n m}\right)-\vec{r}_{n m}\left(\vec{\chi}_{n m} \cdot \vec{\chi}_{m n}\right)\right]} \\
& \dot{\vec{\chi}}_{n m}-\mu \omega \hat{k} \wedge \vec{\chi}_{n m} \\
& =\frac{-\vec{\chi}_{n m}\left(\dot{\vec{r}}_{n m} \cdot \vec{r}_{n m}\right)-\dot{\vec{r}}_{n m}\left(\vec{\chi}_{n m} \cdot \vec{r}_{n m}\right)+\vec{r}_{n m}\left(\vec{\chi}_{n m} \cdot \dot{\vec{r}}_{n m}\right)}{r_{n m}^{2}} \\
& -\sum_{\ell=1 ; \ell \neq m, n}^{N}\left[\frac{\vec{\chi}_{n \ell}\left(\vec{\chi}_{\ell m} \cdot \vec{r}_{n \ell}\right)+\vec{\chi}_{\ell m}\left(\vec{\chi}_{n \ell} \cdot \vec{r}_{n \ell}\right)-\vec{r}_{n \ell}\left(\vec{\chi}_{n \ell} \cdot \vec{\chi}_{\ell m}\right)}{r_{n \ell}^{2}}\right. \\
& \left.+\frac{\vec{\chi}_{n \ell}\left(\vec{\chi}_{\ell m} \cdot \vec{r}_{m \ell}\right)+\vec{\chi}_{\ell m}\left(\vec{\chi}_{n \ell} \cdot \vec{r}_{m \ell}\right)-\vec{r}_{m \ell}\left(\vec{\chi}_{n \ell} \cdot \vec{\chi}_{\ell m}\right)}{r_{m \ell}^{2}}\right] \\
& +\left[f_{n}^{(1)}-f_{m}^{(1)}+\left(f_{n}^{(2)}-f_{m}^{(2)}\right) \hat{k} \wedge\right] \vec{\chi}_{n m}, \\
& n \neq m \text {. }
\end{aligned}
$$

Here the auxiliary dependent variables are the $N(N-1)$ two-vectors $\vec{\chi}_{n m} \equiv \vec{\chi}_{n m}(t)$.

The equations of motion of the third of these models read

$$
\begin{gathered}
\ddot{\vec{r}}_{n}-\omega \hat{k} \wedge \dot{\vec{r}}_{n}=2 \sum_{m=1, m \neq n}^{N} r_{n m}^{-2} . \\
\cdot\left[\left(u_{n m}^{(1)} u_{m n}^{(1)}-u_{n m}^{(2)} u_{m n}^{(2)}\right)+\left(u_{n m}^{(1)} u_{m n}^{(2)}+u_{n m}^{(2)} u_{m n}^{(1)}\right) \hat{k} \wedge\right] \\
\left.\dot{\vec{r}}_{n}\left(\dot{\vec{r}}_{m} \cdot \vec{r}_{n m}\right)+\dot{\vec{r}}_{m}\left(\dot{\vec{r}}_{n} \cdot \vec{r}_{n m}\right)-\vec{r}_{n m}\left(\dot{\vec{r}}_{n} \cdot \dot{\vec{r}}_{m}\right)\right], \\
\dot{u}_{n m}^{(j)}=\sum_{\ell=1 ; \ell \neq m, n}^{N}\left\{\left[r_{n \ell}^{-2}\left\{W_{n \ell m}^{(j)}\left(\dot{\vec{r}}_{\ell} \cdot \vec{r}_{n \ell}\right)+(-)^{j} W_{n \ell m}^{(j+1)}\left[\dot{\vec{r}}_{\ell} \cdot\left(\hat{k} \wedge \vec{r}_{n \ell}\right)\right]\right\}\right]\right. \\
\left.-\left[r_{m \ell}^{-2}\left\{\tilde{W}_{n \ell m}^{(j)}\left(\dot{\vec{r}}_{\ell} \cdot \vec{r}_{m \ell}\right)+(-)^{j} \tilde{W}_{n \ell m}^{(j+1)}\left[\dot{\vec{r}}_{\ell} \cdot\left(\hat{k} \wedge \vec{r}_{m \ell}\right)\right]\right\}\right]\right\} \\
+\left[u_{n m}^{(j)}\left(U_{n m n}^{(1)}-1\right)-u_{n m}^{(2)} U_{n m n}^{(j+1)}\right]\left(\dot{\vec{r}}_{n m} \cdot \vec{r}_{n m}\right) \\
+(-)^{j}\left[u_{n m}^{(j+1)}\left(U_{n m n}^{(1)}-1\right)+u_{n m}^{(j)} U_{n m n}^{(2)}\right]\left[\dot{\vec{r}}_{n m} \cdot\left(\hat{k}_{n} \wedge \vec{r}_{n m}\right)\right] \\
+u_{n m}^{(j)}\left(f_{n}^{(1)}-f_{m}^{(1)}\right)+(-)^{j} u_{n m}^{(j+1)}\left(f_{n}^{(2)}-f_{m}^{(2)}\right), \\
n \neq m, \quad j=1,2 \quad \bmod (2),(1.21 \mathrm{~b})
\end{gathered}
$$

where

$$
W_{n \ell m}^{(j)}=U_{n \ell m}^{(j)}-U_{n \ell n}^{(1)} u_{n m}^{(j)}-(-)^{j} U_{n \ell n}^{(2)} u_{n m}^{(j+1)}, \quad n \neq m, \quad j=1,2 \quad \bmod (2),(1.21 \mathrm{c})
$$




$$
\begin{gathered}
\tilde{W}_{n \ell m}^{(j)}=U_{n \ell m}^{(j)}-U_{m \ell m}^{(1)} u_{n m}^{(j)}-(-)^{j} U_{m \ell m}^{(2)} u_{n m}^{(j+1)}, \quad n \neq m, \quad j=1,2 \quad \bmod (2) \\
U_{n \ell m}^{(j)}=u_{n \ell}^{(1)} u_{\ell m}^{(j)}+(-)^{j} u_{n \ell}^{(2)} u_{\ell m}^{(j+1)}, \quad n \neq m, \quad j=1,2 \quad \bmod (2) .
\end{gathered}
$$

Here the $2 N(N-1)$ auxiliary variables $u_{n m}^{(1)} \equiv u_{n m}^{(1)}(t)$ and $u_{n m}^{(2)} \equiv u_{n m}^{(2)}(t)$ are of course real, and are to be treated as scalars. Note that this many-body problem in the plane is of the (so-called [11] [10] [13] [14]) "goldfish" type, and indeed it clearly admits , when $f_{n}^{(1)}=f_{n}^{(2)}=0$, the reduction $u_{n m}^{(1)}=1, u_{n m}^{(2)}=0$ (entailing $U_{n \ell m}^{(1)}=1, U_{n \ell m}^{(2)}=0$, $\left.W_{n \ell m}^{(1)}=W_{n \ell m}^{(2)}=\tilde{W}_{n \ell m}^{(1)}=\tilde{W}_{n \ell m}^{(2)}=0\right)$, whereby the equations of motion $(1.21 \mathrm{~b})$ are satisfied trivially, while the equations of motion (1.21a) become those of the standard (integrable indeed solvable [8]) "goldfish" model [10].

The equations of motion of the fourth of these models read

$$
\begin{gathered}
\ddot{\vec{r}}_{n}-\omega \hat{k} \wedge \dot{\vec{r}}_{n}=2 \sum_{m=1, m \neq n}^{N} r_{n m}^{-6} . \\
\cdot \vec{V}^{(7)}\left(\dot{\vec{r}}_{n}, \dot{\vec{r}}_{m}, \vec{v}_{n m}, \vec{v}_{m n} ; \vec{r}_{n m}, \vec{r}_{n m}, \vec{r}_{n m}\right), \\
\dot{\vec{v}}_{n m}=\sum_{\ell=1 ; \ell \neq m, n}^{N}\left[\frac{\vec{V}^{(5)}\left(\dot{\vec{r}}_{\ell}, \vec{v}_{n \ell}, \vec{v}_{\ell m} ; \vec{r}_{n l}, \vec{r}_{n l}\right)}{r_{n \ell}^{4}}\right. \\
-\left(\breve{f}_{n}^{(1)}-\breve{f}_{m}^{(1)}\right) \vec{v}_{n m}-\left(\dot{\vec{r}}_{\ell}, \vec{v}_{n \ell}, \vec{v}_{\ell m} ; \vec{r}_{n l}^{(2)}-\vec{r}_{n l}\right) \\
\left.r_{m l}^{4}(2)\right) \hat{k} \wedge \vec{v}_{n m}, \quad n \neq m .
\end{gathered}
$$

Here the auxiliary dependent variables are the $N(N-1)$ two-vectors $\vec{v}_{n m}$, and the two two-vectors $\vec{V}^{(5)}$ respectively $\vec{V}^{(7)}$ are defined in terms of their 5 respectively 7 two-vector arguments in the Appendix, see (A.12) and (A.13). Note that this model might as well be considered of "goldfish type", inasmuch as it features in the right-hand side of (1.22a) "velocity-dependent" forces - but the reduction to the standard "goldfish" model is, in this case, less obvious: it requires a more special choice of the (scalar) source terms $\breve{f}_{n}^{(1)}$ and $\breve{f}_{n}^{(2)}$, as entailed by the treatment of Section 3 (note incidentally the slightly special notation used here for these terms, which is motivated by consistency with the notation used in Section 2, see (2.41c)).

In all the models exhibited above we utilized the (a priori arbitrary, possibly complex) constants $a, b$ when we obtained them in Section 2, but we employed the special assignment (1.7) of these two constants - which entails (see (1.10) and (1.11)) the property of isochronicity, as discussed above and in Section 2 - when writing these models here as 
many-body problems in the horizontal plane. Let us now mention that another remarkable case obtains if the assignment (1.7) is replaced by

$$
a=-i \omega+\gamma, \quad b=0,
$$

with $\gamma>0$ (this correspond to (1.7) with $\mu=1$ and $\omega$ replaced by $\omega+i \gamma$ ). The only modification on the equations of motion entailed by this change is that the left-hand sides of all the first sets of equation, (1.14a), (1.18), (1.19a), (1.20a), (1.21a), (1.22a), then read $\ddot{\vec{r}}_{n}-\omega \hat{k} \wedge \dot{\vec{r}}_{n}+\gamma \dot{\vec{r}}_{n}$, featuring the additional term $\gamma \dot{\vec{r}}_{n}$

clearly interpretable as a friction force, while the left-hand side of (1.14b) reads $\dot{\vec{\rho}}_{n m}-$ $\frac{1}{2} \omega \hat{k} \wedge \vec{\rho}_{n m}+\frac{1}{2} \gamma \vec{\rho}_{n m}$, the left-hand side of $(1.19 \mathrm{~b})$ reads $\dot{\lambda}_{n m}^{(1)}+2 \frac{\hat{k} \cdot \dot{\vec{r}}_{n m} \wedge \vec{r}_{n m}}{r_{n m}^{2}} \lambda_{n m}^{(2)}+$ $\omega \lambda_{n m}^{(2)}-\gamma \lambda_{n m}^{(1)}$ and that of (1.19c) reads $\dot{\lambda}_{n m}^{(2)}-2 \frac{\hat{k} \cdot \dot{\vec{r}}_{n m} \wedge \vec{r}_{n m}}{r_{n m}^{2}} \lambda_{n m}^{(1)}-\omega \lambda_{n m}^{(1)}-\gamma v_{n m}^{(2)}$, the left hand side of (1.20b) reads $\dot{\vec{\chi}}_{n m}-\omega \hat{k} \wedge \vec{\chi}_{n m}+\gamma \vec{\chi}_{n m}$, and the left-hand sides of (1.21b) and $(1.22 \mathrm{~b})$ are unchanged. And it is easily seen from the solution reported in Section 2 that, in this case, the (complex) coordinates $z_{n}(t)$ - related to the real two-vectors $\vec{r}_{n}(t)$ by the standard formulas (3.49) - spiral asymptotically, as $t \rightarrow \infty$, toward finite values $z_{n}(\infty)$ which are the $N$ (complex) eigenvalues of the $N \otimes N$ matrix $X(\infty)$ given by the following explicit formula in terms of the initial data:

$$
\begin{aligned}
& {[X(\infty)]_{n n}=z_{n}(0)+\frac{i}{\omega} \dot{z}_{n}(0),} \\
& {[X(\infty)]_{n m}=-\left[z_{n}(0)-z_{m}(0)\right] M_{n m}(0), \quad n \neq m,}
\end{aligned}
$$

where the initial off-diagonal values $M_{n m}(0)$ are given in terms of the initial values of the auxiliary variables by the simple explicit expressions entailed by the results of Sections 2 and 3 , see (2.36), (2.38), (2.40), (3.51), (3.55), (3.57), (3.59), (3.60).

Some considerations about these results and about prospects of future work - including a terse discussion of the Hamiltonian character of these models - are proffered in Section 4, and several identities relevant to the transition from complex numbers to real two-vectors are reported in the Appendix.

\section{Derivation and solution of the basic dynamical system}

In this section we review the manner to arrive at a dynamical system, which can then be specialized, as shown below, to yield the various models reported above. We thereby ascertain the technique to find the general solution to the initial-value problem of all these models.

Let us take as starting point the linear matrix evolution equation

$$
\ddot{X}+a \dot{X}+b X=0,
$$

where $X \equiv X(t)$ is the evolving $(N \otimes N)$-matrix and $a, b$ are two arbitrary scalar constants. The general solution of the initial-value problem for this equation is of course explicitly known:

$$
X(t)=\exp (\alpha t)\left\{X(0) \cos (\beta t)+\beta^{-1}[\dot{X}(0)-\alpha X(0)] \sin (\beta t)\right\}
$$


with

$$
\alpha=-\frac{a}{2}, \quad \beta=\sqrt{b-\frac{a^{2}}{4}} .
$$

Next, introduce the diagonal $(N \otimes N)$-matrix featuring the eigenvalues - which we call $z_{n}(t)$ - of the matrix $X(t)$ :

$$
\begin{aligned}
& Z(t)=\operatorname{diag}\left[z_{n}(t)\right], \\
& X(t)=R(t) Z(t)[R(t)]^{-1} .
\end{aligned}
$$

Given the matrix $X(t)$, the evaluation of its eigenvalues $z_{n}(t)$ - namely of the diagonal matrix $Z(t)$, see $(2.26)$ - is a purely algebraic task, as well as the evaluation of the diagonalizing matrix $R(t)$, which is however defined up to an arbitrary diagonal matrix $D(t)$,

$$
D(t)=\operatorname{diag}\left[d_{n}(t)\right],
$$

according to the formula

$$
R(t)=\tilde{R}(t) D(t)
$$

since clearly it makes no difference to use $R(t)$ or $\tilde{R}(t)$ in $(2.26 \mathrm{~b})$.

It is now convenient (see below) to introduce the $(N \otimes N)$-matrix

$$
M(t)=[R(t)]^{-1} \dot{R}(t) .
$$

And let us note that the freedom of choice $(2.27 \mathrm{~b})$ gets reflected in the gauge freedom for the matrix $M(t)$ represented - in self-evident notation - by the formula

$$
M(t)=[D(t)]^{-1} \quad \tilde{M}(t) D(t)+[D(t)]^{-1} \dot{D}(t),
$$

where of course

$$
\tilde{M}(t)=[\tilde{R}(t)]^{-1} \dot{\tilde{R}}(t) .
$$

Here and below the matrix $\tilde{R}(t)$, see $(2.27)$, denotes any matrix that diagonalizes the matrix $X(t), X(t)=\tilde{R}(t) Z(t)[\tilde{R}(t)]^{-1}$, see (2.26) - including, say, the "most natural" one that emerges from the diagonalization process.

Note that, via an appropriate assignment of the diagonal matrix $D(t)$, one can always cause the matrix $M(t)$ to have preassigned diagonal elements, say $f_{n}(t)$, since the diagonal part of the matrix formula $(2.29 \mathrm{a})$ reads

$$
f_{n}(t) \equiv M_{n n}(t)=\tilde{M}_{n n}(t)+\frac{\dot{d}_{n}(t)}{d_{n}(t)} .
$$

Let us now see what are the evolution equations satisfied by the eigenvalues $z_{n}(t)$ of the matrix $X(t)$ and by the (off-diagonal) elements of the matrix $M(t)$, entailed by the 
evolution equation (2.25a) satisfied by the matrix $X(t)$. To this end we time-differentiate (2.26b), getting, via (2.28), firstly

$$
\dot{X}=R\{\dot{Z}-[Z, M]\} R^{-1},
$$

and then

$$
\ddot{X}=R\{\ddot{Z}-2[\dot{Z}, M]-[Z, \dot{M}]+[[Z, M], M]\} R^{-1} .
$$

Here and throughout we use of course the standard notation for the commutator of two matrices, $\left[M^{(1)}, M^{(2)}\right] \equiv M^{(1)} M^{(2)}-M^{(2)} M^{(1)}$. Hence from equation (2.25a) we get

$$
\begin{aligned}
\ddot{z}_{n}+a & \dot{z}_{n}+b z_{n}=-2 \sum_{m=1, m \neq n}^{N} M_{n m} M_{m n}\left(z_{n}-z_{m}\right), \\
& \left(z_{n}-z_{m}\right) \dot{M}_{n m}+2\left(\dot{z}_{n}-\dot{z}_{m}\right) M_{n m}+a\left(z_{n}-z_{m}\right) M_{n m} \\
= & \sum_{\ell=1 ; \ell \neq m, n}^{N}\left(z_{n}+z_{m}-2 z_{\ell}\right) M_{n \ell} M_{\ell m}+\left(z_{n}-z_{m}\right) M_{n m}\left(f_{n}-f_{m}\right), \\
n \neq & m,
\end{aligned}
$$

where we have written separately the diagonal and off-diagonal parts of this matrix equation, denoting as $f_{n}$ the diagonal elements of the matrix $M$, see $(2.29 \mathrm{c})$.

This is the basic dynamical system, which will now be presented under various convenient avatars (still others might, of course, be envisaged). But before doing so, let us emphasize that, in these equations of motion, (2.32), we consider the quantities $f_{n}(t)$ as "source terms", which can be assigned as (arbitrarily) given functions of the independent variable $t$, or as (appropriately) given functions of the other dependent variables, $z_{n}$ and $M_{n m}$. We shall occasionally take advantage of this freedom below. Note however that (as already remarked above) the presence of the quantities $f_{n}(t)$ in these equations of motion (2.32) is relatively redundant, since via the position

$$
M_{n m}(t)=\check{M}_{n m}(t) \exp \left[F_{n}(t)-F_{m}(t)\right]
$$

with

$$
\dot{F}_{n}(t)=f_{n}(t)
$$

they get reformulated in completely analogous form, except for the elimination of the $f_{n}$ terms:

$$
\begin{aligned}
\ddot{z}_{n}+a \dot{z}_{n}+b z_{n}=-2 \sum_{m=1, m \neq n}^{N} \check{M}_{n m} \check{M}_{m n}\left(z_{n}-z_{m}\right), \\
\quad\left(z_{n}-z_{m}\right) \dot{M}_{n m}+2\left(\dot{z}_{n}-\dot{z}_{m}\right) \check{M}_{n m}+a\left(z_{n}-z_{m}\right) \check{M}_{n m} \\
=\sum_{\ell=1 ; \ell \neq m, n}^{N}\left(z_{n}+z_{m}-2 z_{\ell}\right) \check{M}_{n \ell} \check{M}_{\ell m}, \quad n \neq m .
\end{aligned}
$$


It is nevertheless convenient to continue to use the version (2.32) of these equations of motion.

The first avatar of the equations of motion (2.32) is obtained via the introduction of the auxiliary dependent variables $g_{n m}$ defined as follows:

$$
M_{n m}(t)=\left[z_{n}(t)-z_{m}(t)\right]^{-2} g_{n m}(t), \quad n \neq m .
$$

We thereby get the equations of motion (1.2).

The second avatar of the equations of motion (2.32) is obtained via the introduction of the auxiliary dependent variables $\gamma_{n m}$ defined as follows:

$$
M_{n m}(t)=\left[z_{n}(t)-z_{m}(t)\right]^{-1} \gamma_{n m}(t), \quad n \neq m .
$$

We thereby get the following equations of motion

$$
\begin{aligned}
\ddot{z}_{n}+a \dot{z}_{n}+b z_{n}=2 \sum_{m=1, m \neq n}^{N} \frac{\gamma_{n m} \gamma_{m n}}{\left(z_{n}-z_{m}\right)}, \\
\dot{\gamma}_{n m}+\gamma_{n m} \frac{\dot{z}_{n}-\dot{z}_{m}}{z_{n}-z_{m}}+a \gamma_{n m} \\
=-\sum_{\ell=1 ; \ell \neq m, n}^{N} \gamma_{n \ell} \gamma_{\ell m}\left(\frac{1}{z_{n}-z_{\ell}}+\frac{1}{z_{m}-z_{\ell}}\right)+\gamma_{n m}\left(f_{n}-f_{m}\right), \\
n \neq m .
\end{aligned}
$$

The third avatar of the equations of motion (2.32) is obtained via the introduction of the auxiliary dependent variables $u_{n m}$ defined as follows:

$$
M_{n m}(t)=-\sqrt{\dot{z}_{n}(t) \dot{z}_{m}(t)}\left[z_{n}(t)-z_{m}(t)\right]^{-1} u_{n m}(t), \quad n \neq m .
$$

Note the introduction in the right-hand side of the square-root term (the appropriateness of this choice can be traced to [6]). We thereby get the following equations of motion:

$$
\begin{aligned}
\ddot{z}_{n}+a \dot{z}_{n} & =2 \sum_{m=1, m \neq n}^{N} \frac{\dot{z}_{n} \dot{z}_{m} u_{n m} u_{m n}}{\left(z_{n}-z_{m}\right)}, \\
\dot{u}_{n m}= & \sum_{\ell=1 ; \ell \neq m, n}^{N} \dot{z}_{\ell}\left[\frac{u_{n \ell}\left(u_{\ell m}-u_{\ell n} u_{n m}\right)}{z_{n}-z_{\ell}}+\frac{u_{\ell m}\left(u_{n \ell}-u_{n m} u_{m \ell}\right)}{z_{m}-z_{\ell}}\right] \\
& +u_{n m}\left[\frac{\left(\dot{z}_{n}-\dot{z}_{m}\right)\left(u_{n m} u_{m n}-1\right)}{z_{n}-z_{m}}+f_{n}-f_{m}\right], \quad n \neq m .
\end{aligned}
$$

To get these equations we did set, for simplicity, $b=0$; and note that, to get $(2.39 \mathrm{~b})$, we also used (2.39a).

The fourth and last avatar of the equations of motion (2.32) that we exhibit here is obtained via the introduction of the auxiliary dependent variables $\eta_{n m}$ defined as follows:

$$
M_{n m}(t)=\sqrt{\dot{z}_{n}(t) \dot{z}_{m}(t)}\left[z_{n}(t)-z_{m}(t)\right]^{-2} \eta_{n m}(t), \quad n \neq m .
$$


(Note again the square-root term in the right-hand side). We thereby get the following equations of motion:

$$
\begin{aligned}
& \ddot{z}_{n}+a \dot{z}_{n}=-2 \sum_{m=1, m \neq n}^{N} \frac{\dot{z}_{n} \dot{z}_{m} \eta_{n m} \eta_{m n}}{\left(z_{n}-z_{m}\right)^{3}} \\
& \dot{\eta}_{n m}=\sum_{\ell=1 ; \ell \neq m, n}^{N} \dot{z}_{\ell} \eta_{n \ell} \eta_{\ell m}\left[\frac{1}{\left(z_{n}-z_{\ell}\right)^{2}}-\frac{1}{\left(z_{m}-z_{\ell}\right)^{2}}\right] \\
& -\eta_{n m}\left[f_{n}-\sum_{\ell=1, \ell \neq n}^{N} \frac{\dot{z}_{\ell} \eta_{n \ell} \eta_{\ell n}}{\left(z_{n}-z_{\ell}\right)^{3}}-f_{m}+\sum_{\ell=1, \ell \neq m}^{N} \frac{\dot{z}_{\ell} \eta_{m \ell} \eta_{\ell m}}{\left(z_{m}-z_{\ell}\right)^{3}}\right] \text {, } \\
& n \neq m \text {. }
\end{aligned}
$$

Again, to get these equations we did set, for simplicity, $b=0$, and to get (2.41b) we also used (2.41a). To get a neater version of these equations of motion, we now set

$$
f_{n}=\breve{f}_{n}+\sum_{\ell=1, \ell \neq n}^{N} \frac{\dot{z}_{\ell} \eta_{n \ell} \eta_{\ell n}}{\left(z_{n}-z_{\ell}\right)^{3}},
$$

so that the second set, (2.41b), of these equations read

$$
\begin{array}{r}
\dot{\eta}_{n m}=\sum_{\ell=1 ; \ell \neq m, n}^{N} \dot{z}_{\ell} \eta_{n \ell} \eta_{\ell m} \\
-\eta_{n m}\left(\frac{1}{\left(z_{n}-z_{\ell}\right)^{2}}-\frac{1}{\left(z_{m}-\breve{f}_{\ell}\right)^{2}}\right], \quad n \neq m .
\end{array}
$$

Let us now indicate, step-by-step, how the initial-value problem for these various equations of motion can be solved.

The first step is to obtain the initial data for the matrix $X(t)$. The relevant formulas read

$$
\begin{aligned}
& X(0)=\operatorname{diag}\left[z_{n}(0)\right], \quad[X(0)]_{n m}=\delta_{n m} z_{n}(0), \\
& {[\dot{X}(0)]_{n n}=\dot{z}_{n}(0),} \\
& {[\dot{X}(0)]_{n m}=-\left[z_{n}(0)-z_{m}(0)\right] M_{n m}(0), \quad n \neq m .}
\end{aligned}
$$

These formulas follow clearly from (2.26b) and (2.30), thanks to the assignment

$$
R(0)=\mathbf{1}, \quad[R(0)]_{n m}=\delta_{n m},
$$

which of course implies that we assume the matrix $X$ to be initially diagonal, see (2.42). Note that this assignment, (2.44), is permissible, and that it still leaves the freedom to assign the initial value $M(0)$ of the matrix $M(t)$, which is just given by the time-derivative of the matrix $R(t)$ at $t=0, M(0)=\dot{R}(0)$ (see (2.28) and (2.44)). Of course, for the various 
avatars of the equations of motion displayed above, the initial values of the off-diagonal elements of the matrix $M(t)$, namely $M_{n m}(0)$ with $n \neq m$, are given in terms of the relevant initial data for the auxiliary variables by the appropriate relations, see (2.35), (2.36), (2.38), (2.40).

The second step is to obtain $X(t)$ from these initial data, and this is accomplished via the explicit formulas (2.25b) with (2.25c). Note that, if the assignment (1.7) of the constants $a, b$ is made - as we indeed generally do - one gets from $(2.25 \mathrm{c})$

$$
\alpha=\frac{i \mu \omega}{2}, \quad \beta=\frac{\omega}{2},
$$

so that $(2.25 \mathrm{~b})$ reads

$$
\begin{aligned}
X(t)= & \exp \left(\frac{i \mu \omega t}{2}\right) . \\
& \cdot\left\{X(0) \cos \left(\frac{\omega t}{2}\right)+\left[\left(\frac{2}{\omega}\right) \dot{X}(0)-i \mu X(0)\right] \sin \left(\frac{\omega t}{2}\right)\right\} .
\end{aligned}
$$

This formula demonstrates that $X(t)$ is in this case periodic in $t$ with period $q T$, see (1.10) and (1.11). Let us also take note of the special assignment

$$
\mu=1, \quad a=-i \omega, \quad b=0
$$

- which might be used in all cases, but must be used in the last two models, see (2.39) and (2.41), characterized by $b=0$. When this special assignment (2.46a) is made, it is interesting to consider the possibility that $\omega$ acquire also a (positive) imaginary part, $\omega \Rightarrow \omega+i \gamma, \gamma>0$, so that (2.45b) become

$$
X(t)=X(0)+\left(\frac{i}{\omega}\right) \dot{X}(0)\{1-\exp [(i \omega-\gamma) t]\},
$$

entailing of course

$$
X(\infty)=X(0)+\left(\frac{i}{\omega}\right) \dot{X}(0) .
$$

The third step is to find the eigenvalues $z_{n}(t)$, see (2.26), of this matrix $X(t)$ : a purely algebraic task. Note that one thereby concludes that the time-evolution of these quantities - the main dependent variables $z_{n}(t)$ - does not depend at all on the "source terms" $f_{n}(t)$ (see $(2.32 \mathrm{~b}),(1.2 \mathrm{~b}),(2.37 \mathrm{~b}),(2.39 \mathrm{~b}),(2.41 \mathrm{~b}))$ or $\breve{f}_{n}(t)$ (see $\left.(2.41 \mathrm{~d})\right)$. This is not surprising, given the possibility to eliminate altogether this type of terms via the transformation (2.33), see (2.34).

The fourth step is to compute a matrix $\tilde{R}(t)$ that diagonalizes the matrix $X(t)$, see (2.26) and (2.27). The ambiguity inherent in the definition of this matrix, see $(2.27 \mathrm{~b})$, shall be taken care of at the end; for the moment any choice of this matrix $\tilde{R}$ is permissible, provided it does diagonalize $X(t)$.

The fifth step is to compute from $\tilde{R}(t)$ the corresponding matrix $\tilde{M}(t)$, see $(2.29 \mathrm{~b})$. 
The sixth step is to compute from $\tilde{M}(t)$ the auxiliary variables $M_{n m}(t)$ via (see $(2.27)$, $(2.28)$ and $(2.29))$

$$
M_{n m}(t)=d_{n}(t) \tilde{M}_{n m}(t)\left[d_{m}(t)\right]^{-1}, \quad n \neq m .
$$

Note that, since the main dependent variables $z_{n}(t)$ are by now known, once the auxiliary dependent variable $M_{n m}(t)$ for the model (2.32) are known, the auxiliary dependent variables featured by the other models - namely $g_{n m}(t)$ (see $\left.(1.2)\right), \gamma_{n m}(t)$ (see $(2.37)$ ), $u_{n m}(t)$ (see $\left.(2.39)\right), \eta_{n m}(t)$ (see $\left.(2.41)\right)$ - are immediately obtained via their relations with the quantities $M_{n m}(t)$, see (2.35), (2.36), (2.38), (2.40). Hence this formula, (2.47), would conclude the task, were it not for the fact that the diagonal elements $d_{n}(t)$ of the matrix $D(t)$ are not yet known.

Hence a seventh step is finally required to complete the job, namely to integrate the ODEs (see $(2.29 \mathrm{c}))$

$$
\dot{d}_{n}=d_{n}\left(f_{n}-\tilde{M}_{n n}\right),
$$

via the quadrature

$$
d_{n}(t)=\exp \left\{\int_{0}^{t} d t^{\prime}\left[f_{n}\left(t^{\prime}\right)-\tilde{M}_{n n}\left(t^{\prime}\right)\right]\right\} .
$$

Here the functions $f_{n}(t)$ are either given as explicit functions of time ("source terms"), or they may be given, in some case (see (2.41c)) as functions of the dependent variables but note that, in all these cases, they can be expressed in terms of the variables $z_{n}(t)$ and $\tilde{M}_{n m}(t)$, for instance $(2.41 \mathrm{c})$ can be clearly re-written as follows (see $(2.40)$ and $\left.(2.47)\right)$ :

$$
f_{n}=\check{f}_{n}+\sum_{\ell=1, \ell \neq n}^{N} \frac{\left(z_{n}-z_{\ell}\right) \tilde{M}_{n \ell} \tilde{M}_{\ell n}}{\dot{z}_{n}} .
$$

This completes the solution - via purely algebraic operations, and a quadrature (to obtain the time-dependent quantities $d_{n}(t)$, see $\left.(2.48 \mathrm{~b})\right)$ - of the initial-value problem for the dynamical systems displayed above, see (2.32), (1.2), (2.37), (2.39), (2.41).

Let us reemphasize in conclusion that, if the assignment (1.7) of the constants $a, b$ (entailing (2.45)) is made, the matrix $X(t)$ evolves periodically in time with period $q T$ (see the sentence after $(2.45 \mathrm{~b})$ ), hence the time evolution of each of its eigenvalues is as well periodic, with a period $\tilde{T}$ which can be at most $N$ ! times larger than $q T$ (due to the possibility that the eigenvalues get reshuffled through the motion). The statement made in the introductory Section 1 about the isochronicity phenomenon is thereby proven. The only nonperiodic solutions are the (nongeneric) ones that become singular at a finite time $t_{c}$, generally due to a collision of two - or, more exceptionally, more than two - of the moving particles (namely they become singular at a time $t_{c}$ - if any - such that $z_{n}\left(t_{c}\right)=$ $z_{m}\left(t_{c}\right)$ for some value of $n$ and $m$ ); for a better understanding of this phenomenology, including its relation to the actual periodicity of the generic (nonsingular) solutions, see the discussion [12] [13] of this mechanism in somewhat analogous situations.

Also note that the results (2.46c) confirm the behavior of certain many-body systems "with friction", as reported at the end of Section 1, see in particular (1.24). 


\section{Real many-body problems in the plane}

In this section we indicate how the (rotation-invariant!) many-body models in the plane reported in Section 1 are related to the various solvable avatars of the main dynamical systems the solution of which is described in the previous Section 2. Some useful identities relevant to this development are confined to the Appendix.

The main trick [9] [10] is to identify appropriately a point, say $z$, in the complex plane, with a real two-vector $\vec{r}$ in a physical plane, which for convenience we identify with the horizontal plane in three-dimensional space. This identification is performed via a correspondence for which we use the notation

$$
z \doteq \vec{r}
$$

the meaning of which is specified by the formulas

$$
z \equiv x+i y, \quad \vec{r} \equiv(x, y, 0), \quad \hat{k} \equiv(0,0,1),
$$

where the components $x, y$ are of course real. In the last of these formulas, (3.49b), we moreover introduce the convenient unit vector $\hat{k}$ orthogonal to the horizontal plane, so that there hold the self-evident relations

$$
i z_{n}=i x-y \doteq \hat{k} \wedge \vec{r}_{n}=(-y, x, 0),
$$

as well as all the others conveniently collected in the Appendix.

This transformation from complex numbers to vectors in the real plane shall now be applied to the avatars of the dynamical system identified in the previous Section 2. It shall, or it shall not - as the case may be, see below - be accompanied by an analogous transformation of complex numbers into real two-vectors for the auxiliary dependent variables featured by each of these models, as displayed in the previous Section 2; the goal is of course to reformulate in every case the equations of motion in covariant form, so that their rotation-invariant character be apparent.

Let us emphasize that, in performing this transformation, we always assume the constants $a$ and $b$ to be assigned according to (1.7), with $\omega$ and $\mu$ both real constants (and $\mu=1$ when $b=0)$, and we set

$$
f_{n}=f_{n}^{(1)}+i f_{n}^{(2)},
$$

with the quantities $f_{n}^{(1)} \equiv f_{n}^{(1)}(t)$ and $f_{n}^{(2)} \equiv f_{n}^{(2)}(t)$ (sometimes decorated with some kind of hat) obviously real and to be always treated as scalars. We moreover often use the short-hand notation (1.16).

The first equations of motion to which we apply this kind of transformation are those of the main dynamical system itself, see (2.32), via the definition

$$
M_{n m}(t)=\mu_{n m}^{(1)}(t)+i \mu_{n m}^{(2)}(t), \quad n \neq m,
$$

where the $2 N(N-1)$ auxiliary dependent variables $\mu_{n m}^{(1)}(t)$ and $\mu_{n m}^{(2)}(t)$ are hereafter treated as real scalars. Then clearly (2.32) become

$$
\begin{array}{r}
\ddot{\vec{r}}_{n}-\mu \omega \hat{k} \wedge \dot{\vec{r}}_{n}+\frac{1}{4}\left(1-\mu^{2}\right) \omega^{2} \vec{r}_{n}=-2 \sum_{m=1, m \neq n}^{N} \\
{\left[\left(\mu_{n m}^{(1)} \mu_{m n}^{(1)}-\mu_{n m}^{(2)} \mu_{m n}^{(2)}\right)+\left(\mu_{n m}^{(1)} \mu_{m n}^{(2)}-\mu_{n m}^{(2)} \mu_{m n}^{(1)}\right) \hat{k} \wedge\right] \vec{r}_{n m},}
\end{array}
$$




$$
\begin{array}{r}
{\left[\dot{\mu}_{n m}^{(1)}+\mu \omega \mu_{n m}^{(2)}+\left(\dot{\mu}_{n m}^{(2)}-\mu \omega \mu_{n m}^{(1)}\right) \hat{k} \wedge\right] \vec{r}_{n m}} \\
+2\left[\mu_{n m}^{(1)}+\mu_{n m}^{(2)} \hat{k} \wedge\right] \dot{\vec{r}}_{n m} \\
=\sum_{\ell=1 ; \ell \neq m, n}^{N}\left[\left(\mu_{n \ell}^{(1)} \mu_{\ell m}^{(1)}-\mu_{n \ell}^{(2)} \mu_{\ell m}^{(2)}\right)+\left(\mu_{n \ell}^{(1)} \mu_{\ell m}^{(2)}-\mu_{n \ell}^{(2)} \mu_{\ell m}^{(1)}\right) \hat{k} \wedge\right]\left(\vec{r}_{n \ell}+\vec{r}_{m \ell}\right) \\
+\left\{\mu_{n m}^{(1)}\left(f_{n}^{(1)}-f_{m}^{(1)}\right)-\mu_{n m}^{(2)}\left(f_{n}^{(2)}-f_{m}^{(2)}\right)\right. \\
\left.+\left[\mu_{n m}^{(1)}\left(f_{n}^{(2)}-f_{m}^{(2)}\right)+\mu_{n m}^{(2)}\left(f_{n}^{(1)}-f_{m}^{(1)}\right)\right] \hat{k} \wedge\right\} \vec{r}_{n m}, \quad n \neq m .
\end{array}
$$

It is now convenient to multiply ("scalar product") the second, (32b), of this set of equations of motion by $\vec{r}_{n m}$ respectively by $\hat{k} \wedge \vec{r}_{n m}$, and to introduce the new (real and scalar) $2 N(N-1)$ auxiliary variables $\lambda_{n m}^{(1)} \equiv \lambda_{n m}^{(1)}(t), \lambda_{n m}^{(2)} \equiv \lambda_{n m}^{(2)}(t)$ by setting

$$
\lambda_{n m}^{(1)}(t)+i \lambda_{n m}^{(2)}(t)=\left[\mu_{n m}^{(1)}(t)+i \mu_{n m}^{(2)}(t)\right] r_{n m}^{2}(t)=M_{n m}(t) r_{n m}^{2}(t), \quad n \neq m .
$$

The equations of motion (3.52) then yield the many-body model in the plane (33).

Next, we transform the avatar (2.37) of the equations of motion, by setting

$$
\gamma_{n m}(t) \doteq \vec{\chi}_{n m}(t)
$$

We thus get (using (A.3) and (A.10)) the equations of motion of the many-body model in the plane (1.20).

The next complex avatar of the equations of motion we now transform into real twovector form is (1.8). To this end we firstly set

$$
g_{n m}=\psi_{n m}^{2}
$$

so that these equations of motion read as follows:

$$
\begin{aligned}
\ddot{z}_{n}-i \mu \omega \dot{z}_{n}+\frac{1}{4}\left(1-\mu^{2}\right) \omega^{2} z_{n}=-2 \sum_{m=1, m \neq n}^{N} \frac{\psi_{n m}^{2} \psi_{m n}^{2}}{\left(z_{n}-z_{m}\right)^{3}} & \\
\dot{\psi}_{n m}-\frac{1}{2} i \mu \omega \psi_{n m}= & -\frac{1}{2} \sum_{\ell=1, \ell \neq m, n}^{N} \frac{\psi_{n \ell}^{2} \psi_{\ell m}^{2}}{\psi_{n m}}\left(\frac{1}{\left(z_{n}-z_{\ell}\right)^{2}}-\frac{1}{\left(z_{m}-z_{\ell}\right)^{2}}\right) \\
& +\frac{1}{2} \psi_{n m}\left(f_{n}-f_{m}\right) .
\end{aligned}
$$

The advantage of this trivial substitution is that these equations are now invariant under the rescaling transformation $z_{n} \Rightarrow c z_{n}, \psi_{n m} \Rightarrow c \psi_{n m}$ where $c$ is an arbitrary constant. Hence [9] [10] we introduce now the real vectors $\vec{\rho}_{n m}$ by setting

$$
\psi_{n m}(t) \doteq \vec{\rho}_{n m}(t), \quad n \neq m
$$


It is then easily seen (via the relevant identities reported in the Appendix) that, via this position (3.57), the equations of motion (3.56) become the equations (1.14), and likewise that (1.9) becomes (1.15) while (1.6a) becomes

$$
\begin{aligned}
& f_{n}^{(1)}=\breve{f}_{n}^{(1)}+\sum_{\ell=1, \ell \neq n}^{N} \frac{2\left(\vec{r}_{n \ell} \cdot \vec{\rho}_{n \ell}\right)^{2}-r_{n \ell}^{2} \rho_{n \ell}^{2}}{r_{n \ell}^{4}}, \\
& f_{n}^{(2)}=\breve{f}_{n}^{(2)}+2 \sum_{\ell=1, \ell \neq n}^{N} \frac{\left(\vec{r}_{n \ell} \cdot \vec{\rho}_{n \ell}\right)\left(\hat{k} \cdot \vec{r}_{n \ell} \wedge \vec{\rho}_{n \ell}\right)}{r_{n \ell}^{4}} .
\end{aligned}
$$

The next complex avatar of the equations of motion we transform into real two-vector form is (2.39). To this end we set (see (2.38))

$$
u_{n m}(t)=u_{n m}^{(1)}(t)+i u_{n m}^{(2)}(t)=-\frac{z_{n}(t)-z_{m}(t)}{\sqrt{\dot{z}_{n}(t) \dot{z}_{m}(t)}} M_{n m}(t), \quad n \neq m
$$

with the proviso to treat the $2 N(N-1)$ auxiliary variables $u_{n m}^{(1)}(t)$ and $u_{n m}^{(2)}(t)$ as real scalars. We thus get the equations of motion of the many-body model in the plane (1.21).

The last complex avatar of the equations of motion we transform here into real twovector form is $(2.41 \mathrm{a})$ with $(2.41 \mathrm{~d})$. To this end we set

$$
\eta_{n m}(t) \doteq \vec{v}_{n m}(t), \quad n \neq m
$$

and we thus get (see the Appendix) the equations of motion of the many-body problem in the plane (1.22).

\section{Outlook}

We did not mention, up to now, the Hamiltonian character of the models investigated herein. Let us just mention now that, following Wojciechowski [23] [24], Nekrasov [20] as well as Babelon and Talon [2], it is easily seen that the equations of motion of the model (1.2) with $a=0$ are produced by the following Hamiltonian $H$ and Poisson brackets $\{\cdot, \cdot\}$ :

$$
\begin{gathered}
H\left(p_{m}, z_{m}, g_{j k} ; t\right)=\frac{1}{2} \sum_{n=1}^{N}\left(p_{n}^{2}+b z_{n}^{2}\right)+\frac{1}{2} \sum_{n, m=1}^{N} \frac{g_{n m} g_{m n}}{\left(z_{n}-z_{m}\right)^{2}}-\sum_{n=1}^{N} f_{n}(t) g_{n n}, \\
\left\{p_{n}, z_{m}\right\}=\delta_{n m}, \quad\left\{p_{n}, g_{m k}\right\}=\left\{z_{n}, g_{m k}\right\}=0 \\
\left\{g_{n m}, g_{k \ell}\right\}=\delta_{n \ell} g_{k m}-\delta_{m k} g_{n \ell} .
\end{gathered}
$$

Indeed it is easily seen that the Hamiltonian equations

$$
\dot{z}_{n}=\left\{H, z_{n}\right\}, \quad \dot{p}_{n}=\left\{H, p_{n}\right\}, \quad \dot{g}_{n m}=\left\{H, g_{n m}\right\},
$$

yield precisely the equations of motion (1.2), and in addition the trivial relations $\dot{g}_{n n}=0$. Note that these ("diagonal") quantities $g_{n n}$ do not appear at all in the equations of motion (1.2), but they do play a role in the Poisson brackets satisfied by the quantities $g_{n m}$ (the 
Poisson character of which is guaranteed by their well known Lie group significance), and their presence in the Hamiltonian (4.1a) is essential to take care of the presence of the "source terms" $f_{n} \equiv f_{n}(t)$ in the equations of motion (1.2) (which is a novelty with respect to the cases treated hitherto [23] [24] [20] [2], and it is the cause of the time-dependence of the Hamiltonian (4.1a)). And let us emphasize that, while the quantities $p_{m}, z_{m}, g_{j k}$ are to be treated as Hamiltonian variables, the quantities $f_{n}$ are instead to be treated as (arbitrarily pre-assigned) "source terms" (which therefore Poisson-commute with all the Hamiltonian variables). This Hamiltonian formulation can be trivially extended to the other models reported in Section 1, such as (1.8) and (1.14), which are directly related to the model (1.2) and do not entail a redefinition of the quantities $f_{n}$ in terms of the Hamiltonian variables. On the other hand the Hamiltonian formulation (if it exists at all) of other models exhibited in Section 1 does not seem to be a trivial matter; we might return to this issue in a future paper.

Also interesting indeed quite amusing - and perhaps worth separate reporting - will be the investigation via numerical simulations of the actual behavior of the solutions of the many-body problems in the plane considered herein.

An open problem is the detailed investigation of the quantum counterpart of the classical many-body problems in the plane considered herein - at least those that allow a Hamiltonian formulation. The conjecture seems plausible that to the isochronous character of the generic solution in the classical case there correspond an equispaced spectrum in the quantum case (at least for some quantization prescription).

Also interesting might perhaps be the investigation of other classical many-body models in the plane obtained via a different complexification [9] [10], that might produce again rotation-invariant models in the plane, although perhaps more artificial ones, lacking the property of translation invariance.

Finally let us mention the possibility - reported separately [15] - to treat in an analogous manner to that used here integrable (indeed solvable, see for instance [10]) models of these types but with more general interactions (say, of hyperbolic rather than rational type).

Acknowledgments. We thank the Institut des Hautes Etudes Scientifiques for the hospitality provided to one of us (FC) for one month in 2003, during which time most of the results reported in this paper were obtained. And we like to acknowledge with thanks several useful exchanges on the topic of this paper with J. Gibbons, J. Hoppe, N. Nekrasov and S. Rauch-Wojciechowski, to all of whom we are particularly indebted for the identification of relevant references. This paper was previously submitted to Annales Henri Poincaré, and after more than one year it was rejected on the basis of a Report by a Referee who had made no effort (or was not capable) to understand what this paper is about and who quite arrogantly stated so: the authors will be happy to send a copy of that Referee report to any interested colleague (it is perhaps time that the behavior of incompetent and arrogant Referees be singled out, as well as that of Editors who condone such behavior). 


\section{Appendix}

\section{A}

In this Appendix we report, for completeness, the definitions and several key identities relevant to the transition from complex numbers such as, say, $z, \zeta$ to real two-vectors in the horizontal plane such as, say, $\vec{r}, \vec{\rho}$, see (3.49).

$$
\begin{aligned}
& z \equiv x+i y \div \vec{r} \equiv(x, y, 0), \quad \zeta \equiv \xi+i \eta \div \vec{\rho} \equiv(\xi, \eta, 0), \quad \hat{k}=(0,0,1), \\
& i z=i x-y \div \hat{k} \wedge \vec{r}=(-y, x, 0), \quad i \zeta=i \xi-\eta \div \hat{k} \wedge \vec{\rho}=(-\eta, \xi, 0), \\
& |z|^{2}=r^{2}, \quad|\zeta|^{2}=\rho^{2} \\
& z \zeta^{*}=(\vec{r} \cdot \vec{\rho})+i[\vec{r} \cdot(\hat{k} \wedge \vec{\rho})] \text {, } \\
& \vec{r} \cdot \vec{\rho}=x \xi+y \eta, \quad \vec{r} \cdot(\hat{k} \wedge \vec{\rho})=x \eta-y \xi ; \\
& \vec{r}=R^{-2}\{\vec{R}(\vec{r} \cdot \vec{R})+\hat{k} \wedge \vec{R}[\vec{r} \cdot(\hat{k} \wedge \vec{R})]\} ; \\
& \left(\hat{k} \wedge \vec{r}_{1}\right)\left[\vec{r}_{2} \cdot\left(\hat{k} \wedge \vec{r}_{3}\right)\right]=\vec{r}_{2}\left(\vec{r}_{1} \cdot \vec{r}_{3}\right)-\vec{r}_{3}\left(\vec{r}_{1} \cdot \vec{r}_{2}\right) \text {, } \\
& {\left[\vec{r}_{1} \cdot\left(\hat{k} \wedge \vec{r}_{2}\right)\right]\left[\vec{r}_{3} \cdot\left(\hat{k} \wedge \vec{r}_{4}\right)\right]=\left(\vec{r}_{1} \cdot \vec{r}_{3}\right)\left(\vec{r}_{2} \cdot \vec{r}_{4}\right)-\left(\vec{r}_{1} \cdot \vec{r}_{4}\right)\left(\vec{r}_{2} \cdot \vec{r}_{3}\right) \text {, }} \\
& \left(\vec{r}_{1} \cdot \vec{r}_{2}\right)\left[\vec{r}_{3} \cdot\left(\hat{k} \wedge \vec{r}_{4}\right)\right]+\left(\vec{r}_{3} \cdot \vec{r}_{4}\right)\left[\vec{r}_{1} \cdot\left(\hat{k} \wedge \vec{r}_{2}\right)\right] \\
& =\left(\vec{r}_{1} \cdot \vec{r}_{4}\right)\left[\vec{r}_{2} \cdot\left(\hat{k} \wedge \vec{r}_{3}\right)\right]+\left(\vec{r}_{2} \cdot \vec{r}_{3}\right)\left[\vec{r}_{1} \cdot\left(\hat{k} \wedge \vec{r}_{4}\right)\right] ; \\
& z_{1} z_{2} \zeta^{*} \div \vec{r}_{1}\left(\vec{r}_{2} \cdot \vec{\rho}\right)+\vec{r}_{2}\left(\vec{r}_{1} \cdot \vec{\rho}\right)-\vec{\rho}\left(\vec{r}_{1} \cdot \vec{r}_{2}\right) \\
& z_{1} z_{2} \zeta^{*} \div R^{-2}\left\{\vec{R}\left[\left(\vec{R} \cdot \vec{r}_{1}\right)\left(\vec{r}_{2} \cdot \vec{\rho}\right)+\left(\vec{R} \cdot \vec{r}_{2}\right)\left(\vec{r}_{1} \cdot \vec{\rho}\right)-(\vec{R} \cdot \vec{\rho})\left(\vec{r}_{1} \cdot \vec{r}_{2}\right)\right]\right. \\
& \left.+\hat{k} \wedge \vec{R}\left\{\left[(\hat{k} \wedge \vec{R}) \cdot \vec{r}_{1}\right]\left(\vec{r}_{2} \cdot \vec{\rho}\right)+\left[(\hat{k} \wedge \vec{R}) \cdot \vec{r}_{2}\right]\left(\vec{r}_{1} \cdot \vec{\rho}\right)-[(\hat{k} \wedge \vec{R}) \cdot \vec{\rho}]\left(\vec{r}_{1} \cdot \vec{r}_{2}\right)\right\}\right\} ; \\
& z_{1} z_{2} \zeta_{1}^{*} \zeta_{2}^{*}=\left(\vec{r}_{1} \cdot \vec{\rho}_{1}\right)\left(\vec{r}_{2} \cdot \vec{\rho}_{2}\right)+\left(\vec{r}_{1} \cdot \vec{\rho}_{2}\right)\left(\vec{r}_{2} \cdot \vec{\rho}_{1}\right)-\left(\vec{r}_{1} \cdot \vec{r}_{2}\right)\left(\vec{\rho}_{1} \cdot \vec{\rho}_{2}\right) \\
& +i\left\{\left(\vec{r}_{1} \cdot \vec{\rho}_{1}\right)\left[\vec{r}_{2} \cdot\left(\hat{k} \wedge \vec{\rho}_{2}\right)\right]+\left(\vec{r}_{2} \cdot \vec{\rho}_{2}\right)\left[\vec{r}_{1} \cdot\left(\hat{k} \wedge \vec{\rho}_{1}\right)\right]\right\} \\
& z_{1} z_{2} z_{3} \zeta_{1}^{*} \zeta_{2}^{*} \doteq \vec{V}^{(5)}\left(\vec{r}_{1}, \vec{r}_{2}, \vec{r}_{3} ; \vec{\rho}_{1}, \vec{\rho}_{2}\right) \\
& =\vec{r}_{3}\left[\left(\vec{r}_{1} \cdot \vec{\rho}_{1}\right)\left(\vec{r}_{2} \cdot \vec{\rho}_{2}\right)+\left(\vec{r}_{1} \cdot \vec{\rho}_{2}\right)\left(\vec{r}_{2} \cdot \vec{\rho}_{1}\right)-\left(\vec{r}_{1} \cdot \vec{r}_{2}\right)\left(\vec{\rho}_{1} \cdot \vec{\rho}_{2}\right)\right] \\
& +\hat{k} \wedge \vec{r}_{3}\left\{\left[\left(\vec{r}_{1} \cdot \vec{\rho}_{1}\right)\left[\vec{r}_{2} \cdot\left(\hat{k} \wedge \vec{\rho}_{2}\right)\right]+\left(\vec{r}_{2} \cdot \vec{\rho}_{2}\right)\left[\vec{r}_{1} \cdot\left(\hat{k} \wedge \vec{\rho}_{1}\right)\right]\right]\right\} \\
& =\vec{r}_{1}\left[-\left(\vec{r}_{2} \cdot \vec{r}_{3}\right)\left(\vec{\rho}_{1} \cdot \vec{\rho}_{2}\right)+\left(\vec{r}_{2} \cdot \vec{\rho}_{1}\right)\left(\vec{r}_{3} \cdot \vec{\rho}_{2}\right)+\left(\vec{r}_{2} \cdot \vec{\rho}_{2}\right)\left(\vec{r}_{3} \cdot \vec{\rho}_{1}\right)\right] \\
& +\vec{r}_{2}\left(\vec{r}_{1} \cdot \vec{\rho}_{1}\right)\left(\vec{r}_{3} \cdot \vec{\rho}_{2}\right)+\vec{r}_{3}\left(\vec{r}_{1} \cdot \vec{\rho}_{2}\right)\left(\vec{r}_{2} \cdot \vec{\rho}_{1}\right) \\
& -\vec{\rho}_{1}\left(\vec{r}_{1} \cdot \vec{r}_{2}\right)\left(\vec{r}_{3} \cdot \vec{\rho}_{2}\right)-\vec{\rho}_{2}\left(\vec{r}_{1} \cdot \vec{r}_{3}\right)\left(\vec{r}_{2} \cdot \vec{\rho}_{1}\right) \text {. }
\end{aligned}
$$


In this last formula, (A.12), the left-hand side is clearly invariant under the exchange of $z_{1}$ with $z_{2}, z_{2}$ with $z_{3}, z_{3}$ with $z_{1}$, or $\zeta_{1}$ with $\zeta_{2}$; this is only partially evident in the expressions in the right-hand sides, but it is of course true as well for all of them.

$$
\begin{aligned}
& z_{1} z_{2} z_{3} z_{4} \zeta_{1}^{*} \zeta_{2}^{*} \zeta_{3}^{*} \\
\doteq & \vec{V}^{(7)}\left(\vec{r}_{1}, \vec{r}_{2}, \vec{r}_{3}, \vec{r}_{4} ; \vec{\rho}_{1}, \vec{\rho}_{2}, \vec{\rho}_{3}\right) \\
= & \vec{r}_{4} \cdot\left\{-\left(\vec{r}_{1} \cdot \vec{\rho}_{3}\right)\left[\left(\vec{r}_{2} \cdot \vec{r}_{3}\right)\left(\vec{\rho}_{1} \cdot \vec{\rho}_{2}\right)+\left(\vec{r}_{2} \cdot \vec{\rho}_{1}\right)\left(\vec{r}_{3} \cdot \vec{\rho}_{2}\right)\right.\right. \\
& \left.+\left(\vec{r}_{2} \cdot \vec{\rho}_{2}\right)\left(\vec{r}_{3} \cdot \vec{\rho}_{1}\right)\right]+\left(\vec{r}_{2} \cdot \vec{\rho}_{3}\right)\left(\vec{r}_{1} \cdot \vec{\rho}_{1}\right)\left(\vec{r}_{3} \cdot \vec{\rho}_{2}\right) \\
& +\left(\vec{r}_{3} \cdot \vec{\rho}_{3}\right)\left(\vec{r}_{1} \cdot \vec{\rho}_{2}\right)\left(\vec{r}_{2} \cdot \vec{\rho}_{1}\right)-\left(\vec{\rho}_{1} \cdot \vec{\rho}_{3}\right)\left(\vec{r}_{1} \cdot \vec{r}_{2}\right)\left(\vec{r}_{3} \cdot \vec{\rho}_{2}\right) \\
& \left.-\left(\vec{\rho}_{2} \cdot \vec{\rho}_{3}\right)\left(\vec{r}_{1} \cdot \vec{r}_{3}\right)\left(\vec{r}_{2} \cdot \vec{\rho}_{1}\right)\right] \\
+\hat{k} \wedge & \vec{r}_{4} \cdot\left\{\left[\left(\vec{r}_{1} \cdot\left(\hat{k} \wedge \vec{\rho}_{3}\right)\right)\right]\left[-\left(\vec{r}_{2} \cdot \vec{r}_{3}\right)\left(\vec{\rho}_{1} \cdot \vec{\rho}_{2}\right)+\left(\vec{r}_{2} \cdot \vec{\rho}_{1}\right)\left(\vec{r}_{3} \cdot \vec{\rho}_{2}\right)\right]\right. \\
+ & \left.\left(\vec{r}_{2} \cdot \vec{\rho}_{2}\right)\left(\vec{r}_{3} \cdot \vec{\rho}_{1}\right)\right]+\left[\vec{r}_{2} \cdot\left(\hat{k}_{1} \wedge \vec{\rho}_{3}\right)\right]\left(\vec{r}_{1} \cdot \vec{\rho}_{1}\right)\left(\vec{r}_{3} \cdot \vec{\rho}_{2}\right) \\
+ & {\left[\vec{r}_{3} \cdot\left(\hat{k} \wedge \vec{\rho}_{3}\right)\right]\left(\vec{r}_{1} \cdot \vec{\rho}_{2}\right)\left(\vec{r}_{2} \cdot \vec{\rho}_{1}\right)-\left[\vec{\rho}_{1} \cdot\left(\hat{k} \wedge \vec{\rho}_{3}\right)\right]\left(\vec{r}_{1} \cdot \vec{r}_{2}\right)\left(\vec{r}_{3} \cdot \vec{\rho}_{2}\right) } \\
- & {\left.\left[\vec{\rho}_{2} \cdot\left(\hat{k} \wedge \vec{\rho}_{3}\right)\right]\left(\vec{r}_{1} \cdot \vec{r}_{3}\right)\left(\vec{r}_{2} \cdot \vec{\rho}_{1}\right)\right\} \cdot }
\end{aligned}
$$

Many other formulas equivalent to those displayed above could as well be written, entailing in particular several other, equivalent, definitions of the two-vectors $\vec{V}^{(5)}\left(\vec{r}_{1}, \vec{r}_{2}, \vec{r}_{3}\right.$; $\vec{\rho}_{1}, \vec{\rho}_{2}$ ) and $\vec{V}^{(7)}\left(\vec{r}_{1}, \vec{r}_{2}, \vec{r}_{3}, \vec{r}_{4} ; \vec{\rho}_{1}, \vec{\rho}_{2}, \vec{\rho}_{3}\right)$ (see (A.12) and (A.13)); in particular from the last formula, (A.13), one can easily get via (A.7) an expression for $\vec{V}^{(7)}\left(\vec{r}_{1}, \vec{r}_{2}\right.$, $\vec{r}_{3}, \vec{r}_{4} ; \vec{\rho}_{1}, \vec{\rho}_{2}, \vec{\rho}_{3}$ ) which does not involve any vector products, and or expressions which appear more symmetrical. And let us finally emphasize that any two-vector can be written as a linear combination with scalar coefficients of an arbitrary two-vector, say $\vec{R}$, and of the two-vector orthogonal to it, $\hat{k} \wedge \vec{R}$, see (A.6) and, for instance, (A.10b).

\section{References}

[1] Arnlind $\mathrm{J}$ and Hoppe J, Eigenvalue-Dynamics off Calogero-Moser, preprint, IHES/P/03/41, 2003.

[2] Babelon O and Talon M, The symplectic structure of the spin Calogero model, preprint arXiv:q-alg/9707011v1.

[3] Barucchi G and RegGe T, Conformal properties of a class of exactly solvable $N$-body problems in space dimension one, J. Math. Phys. 18 (1977), 1149-1153.

[4] Billey E, Avan J and Babelon O, The $r$-matrix structure of the Euler-Calogero-Moser model, preprint arXiv:hep-th/9312042v1.

[5] Billey E, Avan J and Babelon O, Exact Yangian symmetry in the classical EulerCalogero-Moser model, preprint arXiv:hep-th/9401117v1.

[6] Bruschi M and Calogero F, The Lax representation for an integrable class of relativistic dynamical systems, Commun. Math. Phys. 109 (1987), 481-492. 
[7] Calogero F, Exactly Solvable Two Dimensional Many Body Problems, Lett. Nuovo Cimento 16 (1976), 35-38.

[8] Calogero F, Motion of poles and zeros of special solutions of nonlinear and linear partial differential equations, and related "solvable" many-body problems, Nuovo Cimento 43B (1978), 177-241.

[9] Calogero F, Integrable and solvable many-body problems in the plane via complexification, J. Math. Phys. 39 (1998), 5268-5291.

[10] Calogero F, Classical many-body problems amenable to exact treatments, Springer Lecture Notes in Physics Monograph m66, 2001.

[11] Calogero F, The "neatest" many-body problem amenable to exact treatments (a "goldfish"?), Physica D 152-153 (2001), 78-84.

[12] Calogero F and Sommacal M, Periodic solutions of a system of complex ODEs. II. Higher periods, J. Nonlinear Math. Phys. 9 (2002), 483-516.

[13] Calogero F, Françoise J-P and Sommacal M, Periodic solutions of a many-rotator problem in the plane. II. Analysis of various motions, J. Nonlinear Math. Phys. 10 (2003), $157-214$.

[14] Calogero F, Solution of the goldfish N-body problem in the plane with (only) nearestneighbor coupling constants all equal to minus one half, J. Nonlinear Math. Phys. 11 (2004), $102-112$.

[15] Calogero F, A technique to identify solvable dynamical systems, and a solvable generalization of the goldfish many-body problem, J. Math. Phys. 45 (2004), 2266-2279.

[16] Gibbons J and Hermsen T, A generalization of the Calogero-Moser system, Physica D 11 (1984), 337-348.

[17] Krichever I, Babelon O, Billey E and Talon M, Spin Generalization of the CalogeroMoser System and the Matrix KP Equation, Translations of AMS, Series 2, v.170: Advances in Mathematical Sciences, Topics in Topology and Math. Phys., 1995.

[18] Moser J, Three integrable Hamiltonian systems connected with isospectral deformations, Adv. Math. 16 (1975), 197-220.

[19] Nakamura K and Lakshmanan M, Complete integrability in a quantum description of chaotic systems, Phys. Rev. Lett. 57 (1986), 1661-1664.

[20] Nekrasov N, Holomorphic bundles and many body systems, Commun. Math. Phys. 180 (1996), 587-604.

[21] Olshanetsky M A and Perelomov A M, Classical integrable finite-dimensional systems related to Lie algebras, Phys. Rep. 71 (1981), 313-400.

[22] Pechukas P, Distribution of energy eigenvalues in the irregular spectrum, Phys. Rev. Lett. 51 (1983), 943-946.

[23] Wojciechowski S, An integrable marriage of the Euler equations with the Calogero-Moser system, Phys. Lett. A 111 (1984), 101-103.

[24] Wojciechowski S, Generalized integrable many-body systems in one dimension, Phys. Scripta 34 (1986), 304-308. 
[25] Yukawa T, New approach to the statistical properties of energy levels, Phys. Rev. Lett. 54 (1985), 1883-1886.

[26] Yukawa T, Lax form of the quantum mechanical eigenvalue problem, Phys. Lett. A 116 (1986), 227-230. 